\title{
Maturation of Esophageal Motility and Esophagogastric Junction in Preterm Infants
}

\author{
Maissa Rayyan ${ }^{a, b}{\text { Taher Omaric }{ }^{c} \text { Gunnar Naulaers }}^{a, b}$ Raf Aerts $^{d, e}$ \\ Karel Allegaert ${ }^{\mathrm{b}, \mathrm{f}, \mathrm{g}}$ Nathalie Rommel ${ }^{\mathrm{h}-\mathrm{j}}$
}

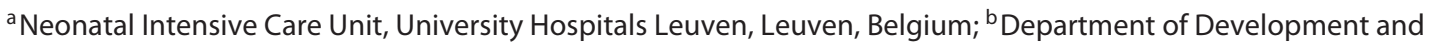
Regeneration, KU Leuven, Leuven, Belgium; ' College of Medicine and Public Health, Flinders University, Adelaide, SA, Australia; ${ }^{d}$ Risk and Health Impact Assessment, Sciensano (Belgian Institute of Health), Brussels, Belgium;

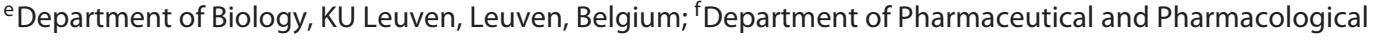
Sciences, KU Leuven, Leuven, Belgium; ${ }^{9}$ Department of Clinical Pharmacy, Erasmus Medical Center, Rotterdam, The Netherlands; ${ }^{h}$ Department of Neurosciences, KU Leuven, Leuven, Belgium; 'Neurogastroenterology and Motility, University Hospitals Leuven, Leuven, Belgium; 'Translational Research Center for Gastrointestinal Disorders (TARGID), Leuven, Belgium

\section{Keywords}

Preterm infant - Maturation · Esophageal motility •

Esophagogastric junction · Lower esophageal sphincter

\begin{abstract}
Background: Preterm infants commonly present with oral feeding problems. The role of maturation of esophageal bolus transport mechanisms herein remains unclear. $\mathbf{O b}$ jectives: To characterize esophageal motility and function of esophagogastric junction (EGJ) during deglutitive swallowing in healthy preterm infants and to describe maturational changes. Methods: Four consecutive high-resolution manometry studies with impedance studies were performed weekly to investigate esophageal motility and EGJ function. Esophageal pressure topography and pressureimpedance metrics were derived. Mixed models with repeated measures were used for statistical analysis. Results: We analyzed 137 nutritive swallows from 36 motility studies in 10 preterm infants. The mean gestational age was
\end{abstract}

$30.17 \pm 0.94$ weeks; the mean postmenstrual age at time point 1 and 4 was $34.42 \pm 0.86$ and $37.45 \pm 1.16$ weeks, respectively. Esophageal peristaltic wave patterns in response to nutritive swallows were observed in all patients. At later time points, esophageal body peristalsis became more rapid, evidenced by a faster distal contractile velocity and shorter distal latency ( $p=0.002$ and $p<0.0001$, respectively). In addition, 4-s integrated relaxation pressures increased and distal contractile integral decreased at later time points ( $p=0.003$ and $p=0.021$, respectively). Bolus clearance also improved at later age $(p=0.008)$. Conclusions: Preterm infants demonstrate peristaltic esophageal motility following nutritive swallows. However, alterations in esophageal bolus transport in relation to peristalsis are demonstrated. Peristaltic progression becomes more rapid, while deglutitive relaxation pressures increase with increasing age. These maturational changes may suggest further development of the enteric nervous system after birth in former preterm neonates.

(c) 2020 S. Karger AG, Basel

\section{KARGER}

(c) 2020 S. Karger AG, Basel 


\section{Introduction}

Deglutition is a complex process. The coordination of rhythmic sequences of sucking, swallowing, and breathing plays a key role in preterm infants [1]. Consequently, problems with swallowing or deglutition in preterm infants are prevalent and can lead to medical complications such as deglutition apneas, desaturation, aspiration, and feeding difficulties [1-3]. These feeding problems are more prevalent in preterm infants with low gestational age because of their immature sucking and swallowing system, neurological and gastrointestinal immaturity, and frequent comorbidities [4-6]. Development of coordination skills followed by efficient esophageal transport is crucial for safe oral feeding [7]. Esophageal motility is a key component in performing successful and complete clearance of a bolus during oral feeding. It is also important for clearance of refluxate. Normal esophageal peristaltic movements and esophagogastric junction (EGJ) function have been well established in adults and children, but normal development of esophageal motility in preterm infants has not yet been fully defined [8]. The main reasons for this lack of information are the semiinvasive nature of the examination with manometry and the ethics of submitting healthy infants to these tests. Despite this limitation, we have recently been able to describe the effect of a shorter esophageal length on esophageal bolus transport metrics in a healthy young infant population [9].

Until now, research on esophageal motility has focused on qualitatively describing esophageal peristaltic patterns following elicited swallowing reflexes by facial stimulation (Santmyer reflex) and spontaneous non-nutritive swallows [10-13]. These swallow-triggered peristaltic patterns appear to be normally propagated, even in very premature infants. Although knowledge on esophageal motility is essential to understand the physiology of normal feeding in preterm infants, motility in response to nutritive swallows remains unexplored using state-ofthe-art objective measures. The EGJ has been well studied during spontaneous swallowing. It is a high-pressure zone at the distal esophagus and consists of three structures: the lower esophageal sphincter (LES), the diaphragmatic crura, and the sling fibers of the gastric cardia. Data on maturational changes of the resting pressure at the LES in function of postmenstrual age, however, are conflicting $[11,14]$. Nonetheless, the LES tone seems capable to act as an antireflux barrier. Transient relaxations of the LES (TLESRs) are, however, the most common mechanism of gastroesophageal reflux in preterm infants [15].
TLESRs occur mainly triggered by gastric distension and are not related to swallowing [16].

Myelination of the nervous system in the cortex and brainstem starts in the third trimester and continues until adolescence. This myelination is essential for impulse conduction and expresses functional maturity [17]. At this point in time, it is unclear whether this increasing myelination plays a role in esophageal function. Hence, it is unclear whether the esophageal function is altered with increasing age. The objectives of this prospective study were to describe esophageal motility during normal feeding, the function of the EGJ in preterm infants, and the maturational changes using objective metrics as a function of age.

\section{Material and Methods}

Informed consent was obtained from all participants' primary caregivers before the start of the study. All studies were performed bedside in the neonatal intensive care unit (NICU) of the University Hospitals Leuven (Belgium).

\section{Patients}

Ten preterm infants admitted to the NICU of the University Hospitals Leuven were included in the study. Only healthy preterm infants weighing $>1,500 \mathrm{~g}$ at the time of the first study were eligible. Patients with associated comorbidities such as bronchopulmonary dysplasia (defined by oxygen need at postnatal age (PNA) 28 days), necrotizing enterocolitis, or any type of intraventricular hemorrhage were excluded from participation as well as infants who were on respiratory support or oxygen therapy. Clinical data on age (gestational age (GA), postmenstrual age (PMA), and PNA), weight, oral feeding intake, or drug use (PPI, H2 blockers, prokinetic drugs, caffeine) were recorded. PMA was calculated by adding PNA to GA [18]. The first manometric study was performed when infants were cardiorespiratory stable and showed readiness for oral feeding. The interval between each study was set to 7 days.

\section{High-Resolution Impedance Manometry Recordings}

Four motility studies with a weekly interval were scheduled. Because of ethical considerations, the studies were only performed in case the infant was still being fed by a nasogastric tube. Highresolution manometry combined with impedance (HRIM) recordings were acquired using an 8-Fr solid state catheter incorporating 13 pressure sensors spaced $1 \mathrm{~cm}$ apart, and 6 adjoining impedance segments, each $2 \mathrm{~cm}$ apart (Unisensor AG, Switzerland). The indwelling nasogastric tube was removed before placement of the catheter. The probe was placed by experienced caregivers (M.R. and N.R.). Guided by a color screen pressure plot in real time, the probe was passed along the esophagus with the tip through the LES and placed in the stomach ensuring that at least 1 pressure and 1 impedance channel was positioned in the stomach. A central lumen in the catheter was used for administration of tube feeding at the end of the study whenever the oral intake of the infant was insufficient. Pressure and impedance data were acquired at 20 samples per second (Solar GI; Medical Measurement Sys- 
Fig. 1. Derivation of esophageal function metrics. Esophageal pressure topography plot during swallowing of a liquid bolus. This Clouse plot is derived from data acquired from high-resolution manometry studies and describes peristaltic amplitudes in a space (vertical axis) and time continuum (horizontal axis) displayed using a color code. The anatomy panel illustrates the anatomical position. The exact time of maximum distension of the esophageal lumen just proximal to the esophagogastric junction (EGJ) is localized by nadir impedance and is indicated by a red asterisk. The following esophageal pressure topography metrics are displayed on the Clouse plot: distal contractile integral (DCI), a marker for contractile vigor; distal latency time (DL), the time interval between UES relaxation and CDP, and EGJ-integrated relaxation pressure (IRP4). IRP4 is the median pressure measured over the EGJ for $4 \mathrm{~s}$ of maximal relaxation after UES relaxation. Bolus latencies are determined by swallow (arrow) to distension (red asterisk) latency (SDL) and distension to contraction latency (DCL). DL is the sum of SDL and DCL. $\mathrm{CD}$, crural diaphragm; CDP, contractile deceleration point; TZ, transition zone; UES, upper esophageal sphincter.

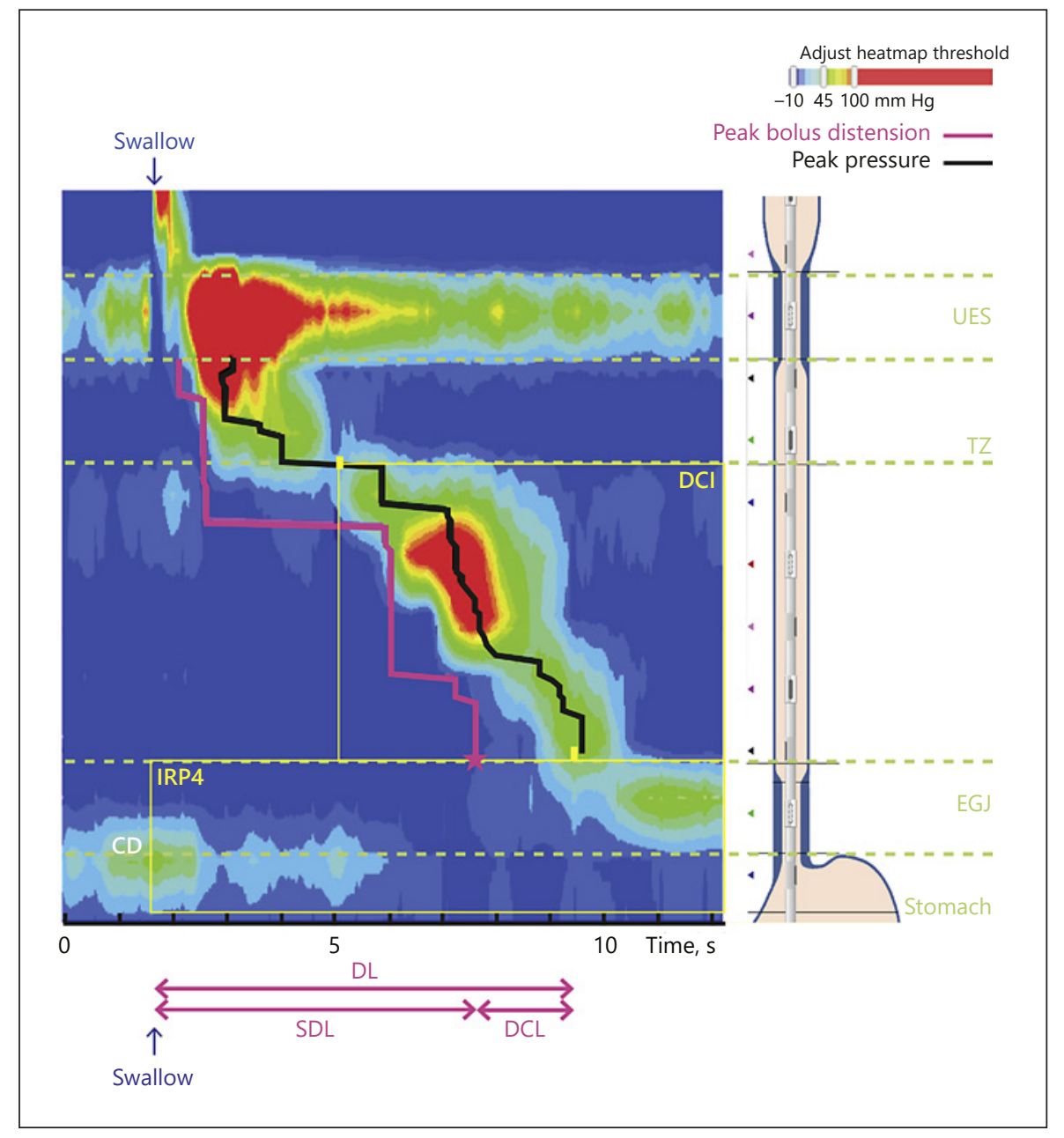

tems, The Netherlands). The infants were monitored using oxygen saturation and ECG monitoring during the whole procedure. Oral sucrose $24 \%$ was used for procedural analgesia before placement of the probe. The lights were dimmed, and a quiet environment was created. The parents were asked to be present to comfort their child with the help of the attending nurse. The infants were fed in their habitual position with a bottle with expressed breastmilk or formula milk. Whenever bottle feeding was not possible, milk boluses of $0.5 \mathrm{~mL}$ were administered orally using a syringe. Bolus conductivity was enhanced for impedance readings by adding a $10 \%$ dilution of $\mathrm{NaCl} 0.9 \%$ to the milk.

\section{HRIM Analysis}

Normal peristaltic wave patterns were defined as the presence of propagating pressure waves after deglutition along the esophagus from the upper esophageal sphincter (UES) to the EGJ [19]. Analysis of the HRIM recordings was applied via a web-based application called Swallow Gateway ${ }^{\mathrm{TM}}$ (available at: swallowgateway. com) as has been recently described [20]. The HRIM study was first exported as ASCII file and subsequently uploaded on the website for further analysis. Only nutritive swallows were selected and analyzed. A nutritive swallow was defined as a swallow followed by an increase in admittance signal at the distal UES margin (indicat- ing bolus flow) and with a bolus trajectory evidenced by an impedance signal along the esophagus (Fig. 1).

Four esophageal pressure topography metrics were extracted: (1) distal contractile integral (DCI), a marker of contractile vigor calculated as the product of amplitude, duration, and span of the distal esophageal contraction; (2) distal contractile velocity (DCV) stands for the velocity of the propagating contraction - a high value indicates rapid contraction -; (3) distal latency (DL) reflects the time interval between UES and contractile deceleration point (CDP), CDP is the inflection point at which propagation velocity in the distal esophagus slows down - this point discriminates the transition from the distal esophagus to the LES -; (4) four-second integrated relaxation pressure (IRP4) is the median EGJ pressure measured during $4 \mathrm{~s}$ of maximal relaxation during a 10 -s period after UES relaxation.

Five additional pressure-impedance-derived metrics were also extracted (Fig. 1): (1) intrabolus distension pressure during esophageal emptying. This is the distension pressure within the bolus domain that precedes smooth muscle peristalsis. Bolus flow latencies are based on pressure and impedance recordings at the CDP being (2) swallow to distension latency (SDL) (measured from swallow to nadir impedance) and (3) distension to contraction latency. DL is the sum of SDL and distension to contraction latency. 
Table 1. Summary of patient characteristics during the four consecutive high-resolution impedance manometry studies

\begin{tabular}{lllll}
\hline & Time point 1 & Time point 2 & Time point 3 & Time point 4 \\
\hline Patients, $n$ & $10(8$ boys $)$ & $10(8$ boys $)$ & $10(8$ boys $)$ & $6(5$ boys $)$ \\
GA, weeks & $30.17 \pm 0.94$ & $30.17 \pm 0.94$ & $30.17 \pm 0.94$ & $30.24 \pm 0.93$ \\
PNA, days & $29.17 \pm 7.9$ & $36.33 \pm 8.5$ & $43.33 \pm 8.2$ & $50.50 \pm 8.3$ \\
PMA, weeks & $34.42 \pm 0.86$ & $35.42 \pm 0.90$ & $36.30 \pm 1.08$ & $37.45 \pm 1.16$ \\
Birth weight, g & $1,233 \pm 264$ & $1,233 \pm 264$ & $1,233 \pm 264$ & $1,137 \pm 265$ \\
Weight at study, g & $1,862 \pm 235$ & $2,150 \pm 241$ & $2,430 \pm 241$ & $2,491 \pm 121$ \\
Caffeine treatment, $n$ & 4 & 0 & 0 & 0 \\
Erythromycin treatment, $n$ & 1 & 1 & 1 & 0 \\
Oral intake, \% of total enteral & $18 \pm 19$ & $40 \pm 26$ & $65 \pm 31$ & $87 \pm 23$ \\
\hline
\end{tabular}

Mean values \pm SD are given. GA, gestational age; PNA, postnatal age; PMA, postmenstrual age (= GA+PNA).

(4) Contractile segment impedance (CSI) is the impedance value at peak esophageal contraction measured at the distal one third above the EGJ margin. CSI can be used as a measure for mucosal integrity $[21,22]$. Finally, (5) luminal clearance was defined by impedance ratio (IR). IR is determined by the ratio of nadir impedance when the esophageal lumen is full and maximally distended and impedance at peak contraction when the esophageal lumen is empty and occluded. A higher IR indicates more bolus residual and, thus, less effective bolus clearance.

For the evaluation of the EGJ barrier function, mean resting pressure and contractile integral (EGJ-CI) were determined. EGJ$\mathrm{CI}$ was measured over a period of 3 respiratory cycles above threshold of gastric pressure [23]. EGJ-CI metric was measured 3 separate times at the beginning of the manometric study. Finally, the length of the esophagus was measured at the start of each study from the lower margin of the UES to the proximal margin of the EGJ.

\section{Statistical Analysis}

The median value for each swallow parameter for each individual case was determined, and a mixed model analysis with repeated measures was used to investigate the time effect. To set up the mixed model analysis, "time" was set as a repeated as well as a fixed variable. A random intercept was included in the model. To account for potential effects of age, GA and PNA at the start of the first study were included as covariates. Bonferroni's confidence interval adjustment was applied. Data are presented as means, estimated marginal means, and standard errors (for the mixed models). In a first instance, an unstructured covariance model was applied. The model was then modified to a compound symmetry and heterogeneous autoregressive (AR1) model. The Akaike information criterion was calculated to estimate the goodness of fit of the model. The model with the lowest Akaike information criterion was withheld and reported.

Statistical data analysis was conducted using IBM SPSS Statistics (released 2019, version 26; IBM Corp, Armonk, NY, USA). A $p$ value $<0.05$ indicated statistical significance for time, GA, and PNA. The reported $p$ values refer to the overall $p$ values of the fully adjusted mixed model. Relation to PMA was assumed for parameters significantly related to GA and PNA. Associations between oral feeding intake and swallow variables were analyzed using Spearman's rank correlation.

\section{Results}

\section{Cohort Characteristics}

Ten healthy preterm infants were included in this study. Each patient was clinically assessed by the principal investigator (M.R.) and the attending neonatologist prior to each HRIM investigation. Patient characteristics are summarized in Table 1. Mean GA at birth was $30.17 \pm 0.94$ weeks, and mean birth weight was $1,233 \pm 264$ g. Six patients underwent four consecutive weekly motility studies. In four patients, the final study was not performed because the infants no longer required supplemental nasogastric feeding. None of the patients received respiratory support or supplemental oxygen at the time of the study. Four patients were treated with caffeine at the time of the first study but treatment was discontinued in all patients at the time of the second study. One patient was treated with erythromycin for clinical suspected gastroesophageal reflux during the first three HRIM studies. The included patients received neither specific therapeutic treatment nor home tube feeding at discharge. A total of 36 HRIM studies were performed, and 137 nutritive swallows were analyzed (total swallows $37,38,38$, and 24 ; mean swallows/patient 3.7, 3.8, 3.8, and 4 at study $1,2,3$, and 4 , respectively). The studies were tolerated well by all patients, and no acute cardiorespiratory events were documented.

\section{Esophageal Motility Parameters}

All included infants displayed qualitatively propagating peristaltic esophageal motor patterns in response to nutritive swallows [19]. Computed parameters during nutritive swallows are summarized in Table 2. The terms "age" and "time" will be interchanged throughout the Results and Discussion sections but not for GA, PNA, or PMA. 
Table 2. Esophageal pressure topography and esophageal pressure-impedance parameters

\begin{tabular}{|c|c|c|c|c|c|c|c|c|}
\hline \multirow[t]{2}{*}{ Parameter } & \multicolumn{4}{|l|}{ Time point } & \multicolumn{3}{|c|}{$\begin{array}{l}p \text { values related to } \\
\text { different factors }\end{array}$} & \multirow[t]{2}{*}{$\begin{array}{l}\text { Relation to } \\
\text { time and age }\end{array}$} \\
\hline & 1 & 2 & 3 & 4 & time & GA & PNA & \\
\hline \multicolumn{9}{|l|}{ Esophageal pressure topography } \\
\hline $\mathrm{DCI}, \mathrm{mm} \mathrm{Hg} \times \mathrm{cm} \times \mathrm{s}$ & $1,064(153)$ & $948(155)$ & $804(116)$ & $556(77)$ & $*$ & NS & NS & Time \\
\hline $\mathrm{DCV}, \mathrm{cm} / \mathrm{s}$ & $1.01(0.05)$ & $1.54(0.14)$ & $1.57(0.12)$ & $1.73(0.26)$ & $* *$ & NS & NS & Time \\
\hline $\mathrm{DL}, \mathrm{s}$ & $7.13(0.30)$ & $5.41(0.30)$ & $4.97(0.30)$ & $4.83(0.38)$ & $* * *$ & NS & NS & Time \\
\hline IRP4, mm Hg & $6.3(2.5)$ & $9.6(2.5)$ & $17.3(2.5)$ & $13.4(3.1)$ & $* *$ & NS & NS & Time \\
\hline EGJ resting pressure, $\mathrm{mm} \mathrm{Hg}$ & $49(14)$ & $41(3)$ & $46(8)$ & $62(9)$ & NS & $*$ & $*$ & $\begin{array}{l}\text { GA, PNA, } \\
P M A\end{array}$ \\
\hline EGJ-CI, mm Hg.cm & $46(13)$ & $37(4)$ & $46(9)$ & $58(9)$ & NS & NS & NS & - \\
\hline Esophageal length, mm & $62.1(1.4)$ & $62.0(1.1)$ & $65.2(2.0)$ & $62.9(1.4)$ & NS & NS & NS & - \\
\hline \multicolumn{9}{|l|}{ Pressure-impedance analysis } \\
\hline DPE, mm Hg & $19(2)$ & $16(1)$ & $21(2)$ & $22(3)$ & NS & NS & NS & - \\
\hline SDL, s & $5.32(0.51)$ & $2.98(0.32)$ & $3.53(0.44)$ & $2.79(0.28)$ & $* *$ & NS & $*$ & Time, PNA \\
\hline DCL, s & $2.33(0.55)$ & $3.14(0.55)$ & $2.15(0.55)$ & $2.85(0.64)$ & NS & NS & NS & - \\
\hline IR & $0.83(0.02)$ & $0.77(0.02)$ & $0.73(0.02)$ & $0.75(0.02)$ & $* *$ & $*$ & $*$ & $\begin{array}{l}\text { Time, GA, } \\
\text { PNA, PMA }\end{array}$ \\
\hline $\mathrm{CSI}, \Omega$ & $750(40)$ & $793(40)$ & $866(40)$ & $916(51)$ & $*$ & $*$ & NS & Time, GA \\
\hline
\end{tabular}

Estimated marginal mean values (SE) are based on mixed model analysis. Marginal means were evaluated at GA 30.2 weeks and at PNA_start first study 29.6 weeks. $p$ values for time, GA, and PNA are based on mixed models ${ }^{\#}$ Relation for PMA was assumed for parameters significantly related to GA and PNA.

GA, gestational age; PNA, postnatal age; PMA, postmenstrual age; DCI, distal contractile integral; DCV, distal contractile velocity; DL, distal latency; IRP4, 4-second integrated relaxation pressure; EGJ resting pressure, resting pressure at the esophagogastric junction; EGJCI, EGJ contractile integral; DPE, distension pressure during esophageal emptying; SDL, swallow distension latency; DCL, distension contraction latency; IR, impedance ratio; CSI, contractile segment impedance.

${ }^{*} p<0.05,{ }^{* *} p<0.01,{ }^{* * *} p<0.001$.

Time had a main effect on DCV, DL, and SDL (DCV time, $p=0.002$; DL time, $p<0.0001$; SDL time, $p=0.004$ ) (Fig. 2a, b). Hence, contraction and bolus arrival at the EGJ was more rapid in infants at later time points. Other effects observed include an increase in IRP4 at later time points (time, $p=0.003$ ). However, the relevance of this finding was unclear as intrabolus distension pressure during esophageal emptying was unaffected (time, $p=$ 0.129 ). Mucosal impedance (CSI) increased (time, $p=$ 0.027 ) causing the IR to decrease at increasing time points (time, $p=0.008$ ). Interestingly, the strength of the esophageal contraction decreased at later time points (DCI time, $p=0.021$ ). DCI was not related to GA nor PNA (GA, $p=0.837$; PNA, $p=0.774)$. EGJ resting pressures seemed to increase related to GA and PNA at the start of study, but not to time (GA, $p=0.018$; PNA, $p=0.029$; time, $p=$ 0.14 ). Higher GA and PNA (hence, PMA) were associated with higher EGJ resting pressures and with lower IR (data not shown).
At each time point, the percentage of oral intake increased for the studied subjects (Table 1). At time point 4 , the mean percentage of oral intake to total enteral was $87 \pm 23 \%$. Four patients were on full oral feeding at time point 4 . Esophageal length did not change with age within this 4-week time interval. We did not find an association between oral feeding intake and the studied swallow function variables (Spearman's rank, NS).

\section{Discussion}

This study characterizes esophageal motility patterns, metrics of esophageal peristaltic, and EGJ barrier function of healthy preterm infants during postnatal maturation. The most important finding of this prospective study is that the speed of peristaltic propagation increases as the infant matures. Consistent with previous reports, the EGJ barrier function is already well established in young preterm infants [11]. 

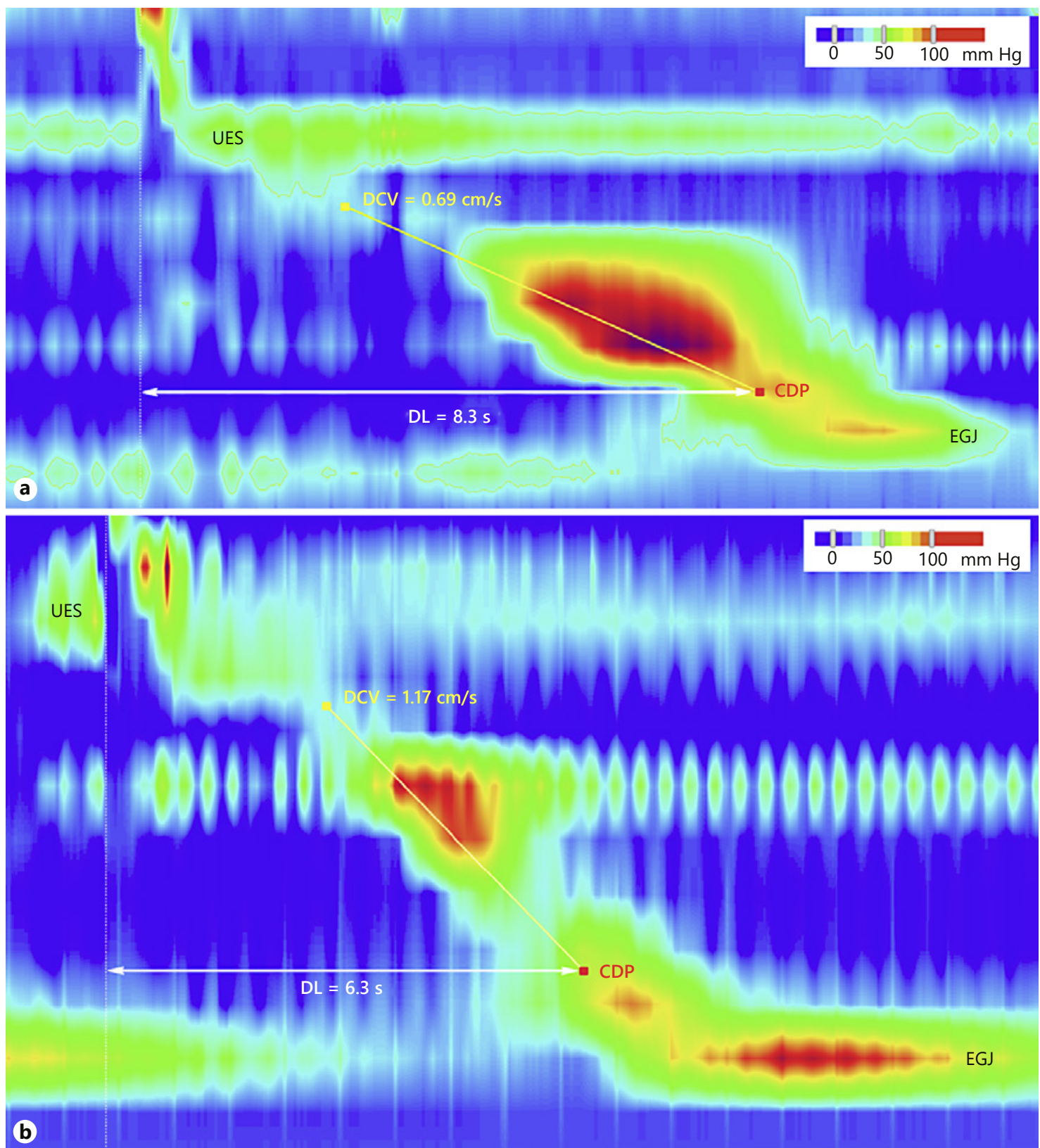

Fig. 2. Illustration of maturation of pressure-flow parameters during nutritive swallow in a preterm study patient at time point 1 (a) and time point 3 (b). Swallows are shown as recorded by highresolution impedance manometry and displayed as esophageal pressure topography plots. Distal contractile velocity (DCV) demonstrates the velocity of the propagating peristaltic wave. Distal latency (DL) is displayed as the time interval between upper sphincter relaxation (UES) and contractile deceleration point (CDP). DCV increases and DL decreases with age. DCV at time point 1: $0.69 \mathrm{~cm} / \mathrm{s}$; at time point 3: $1.17 \mathrm{~cm} / \mathrm{s}$. DL at time point 1 : $8.3 \mathrm{~s}$; at time point 3: $6.3 \mathrm{~s}$. EGJ esophagogastric junction.

propagated contractions in response to dry swallows were seen in almost $30 \%$ of the swallows [10]. In the above-mentioned study, the percentage of peristaltic waves did not change with increasing postnatal age, although other investigators have reported higher rates of completed peristaltic but were confined to dry swallows $[10,13]$. Anterograde 
contractions with increasing age in the second esophageal segment [13]. In the present study, peristaltic contractions were registered in response to nutritive swallows in all investigated patients at weekly sequential time points.

With increasing age, peristalsis propagates faster (increased DCV), and the swallowed bolus arrives earlier (decreased DL) in the distal esophagus. This observation is not related to an increase in esophageal length, although the DL is known to increase with esophageal length in older children [20]. The reason for this decreased DL remains unclear; however, we speculate that bolus propulsion by the tongue base and pharynx may become stronger with increasing age. Alternatively, the subtle changes seen may reflect maturational changes within the enteric nervous system. Propagation rate is known to be influenced by the inhibitory-excitatory balance within the enteric nervous system, whereby a relative reduction in inhibition or, conversely, augmentation of excitation may result in more rapid propagation [24]. We also noted a reduced LES relaxation (increase in IRP4) which could also be indicative of changes related to integrated enteric nervous system mechanisms. Esophageal peristalsis is dependent on central and peripheral vagal pathways. The neurons in the motor and sensory vagal nerve nuclei in the brainstem of preterm infants are immature [25]. More mature neurons are seen in older infants. In addition, the myelination of vagal fibers continues during the first year, particularly the first 3 months of life [26]. These observations suggest that vagal innervation matures in preterm infants with concomitant improvement of conduction velocity and yet improved esophageal motility. The DCI, however, a parameter for esophageal peristaltic vigor, decreases with age. The reason for this decreased DCI remains unclear. Although not measured in this study, we hypothesize that the pharyngeal contraction increases with time, moving the bolus further down into the esophagus. Hence, there is less need for a strong esophageal contraction (DCI) to push the bolus through the EGJ.

Other changes seen with age include an increased mucosal impedance (CSI). In older children and adults, a low CSI has been proposed as a measure loss of mucosal integrity due to gastroesophageal reflux disease and/or eosinophilic esophagitis [27]. In the current study, the CSI increased in infants at later time points which may reflect an improvement of epithelial barrier function with age.

In the past, maturational changes of the LES function have been explored during dry swallows and in response to mid-esophageal provocations [11, 14, 28]. Pena et al. [14] demonstrated shorter response latency to LES relaxation in response to liquid stimulation and longer LES

Esophageal Motility in Healthy Preterms relaxation in relation to age. In the same study, resting LES pressures seemed to increase with age [14]. Other studies have refuted maturational changes of the resting LES pressure and concluded that the LES tone was sufficient to act as an antireflux barrier in very preterm infants $[11,15]$. The data from the current study reinforce the absence of maturational changes in non-deglutitive LES function. Although we did not notice a difference in nondeglutitive LES function in function of time, the data from the current study suggest an increase in EGJ resting pressures related to GA, PNA, and PMA. In this study, however, we did not formally evaluate gastroesophageal reflux episodes or the role of TLESRs.

IRP4 is a manometric parameter used to define median pressure during relaxation of LES after deglutition [29]. IRP4 is not only determined by crural contraction during respiration and relaxation of LES, but also by increased bolus distension pressure in the esophagus [30]. We hypothesize that the increase in IRP4 with age does not necessarily reflect a reduced relaxation of the EGJ, but could rather be influenced by the increase in intrabolus pressure at the EGJ caused by the intake of larger oral volumes [30].

The limitations of this study need to be addressed. First, the data are based on a limited number of patients with a limited number of swallows analyzed per patient. However, we were selective in the collection of swallows for pressureimpedance analysis. We included only larger swallows with clear impedance signals (indicating bolus flow) and propagating peristalsis. Previous research has shown that 4 swallows per patient were sufficient to provide a reliable analysis [31]. These data from a limited number of patients need to be confirmed in a larger number of patients, however, ethical considerations might impede such larger studies. Second, the analysis of nutritive boluses is challenging especially when preterm infants drink in a physiological way using consecutive swallows during bottle feeding. However, we managed to select an adequate number of analyzable swallows leading to the presented results. The administration of isolated oral boluses could facilitate the analysis and interpretation of the esophageal peristaltic contraction but does - on the other hand - not fully reflect the physiological way of drinking of a young infant.

\section{Conclusion}

This prospective study shows maturational changes in esophageal function in preterm infants. With increasing age, the esophageal peristaltic contraction during nutri- 
tive swallowing becomes faster, and the extent of EGJ relaxation reduces. However, we noticed an increased EGJ barrier in preterm infants with higher PNA and PMA. The mechanisms controlling the EGJ barrier in rest, however, were unaffected over the different time points. These maturational changes are important to acknowledge when trying to understand normal esophageal motility after preterm birth.

\section{Statement of Ethics}

This study was approved by the Ethics Committee of the University Hospitals Leuven (Leuven, Belgium). Informed consent was obtained from all participants' primary caregivers before the start of the study.

\section{Disclosure Statement}

Nathalie Rommel and Taher Omari hold a patent on AIMplot, the software used to analyze the pressure flow data. The open access Swallow Gateway ${ }^{\mathrm{TM}}$ resource is provided and hosted by Flinders University. None of the other authors has any potential conflicts of interest to declare.

\section{Funding Sources}

Maissa Rayyan was supported by the fund for clinical research, University Hospitals Leuven.

\section{Author Contributions Statement}

M.R. was the principal investigator for the study. She designed the study, made the application to the Ethics Committee, included all patients, acquired all data, and conducted the data analysis and statistical analysis. She wrote the first draft of the manuscript and revised the manuscript based on reviews by the co-authors. N.R. developed the study design, acquired all data, and oversaw the data analysis. Together with M.R., she had access to all study data and takes responsibility for the integrity of the data and accuracy of data analysis. She reviewed and supervised the finalization of the manuscript. T.O. was critical in further developing the study design and providing technical support on the software analysis. He made a significant contribution by critically reviewing the manuscript. K.A. was involved in the study design and contributed significant intellectual work by critically reviewing the manuscript. G.N. made significant contributions to the manuscript. R.A. helped to carry out the statistical analysis and critically reviewed the manuscript.

All authors approved the final draft and agree to be accountable for all aspects of the work in ensuring that questions related to the accuracy or integrity of any part of the work are appropriately investigated and resolved.

\section{References}

1 Gewolb IH, Vice FL, Schwietzer-Kenney EL, Taciak VL, Bosma JF. Developmental patterns of rhythmic suck and swallow in preterm infants. Dev Med Child Neurol. 2001 Jan;43(1):22-7.

2 Rommel N, De Meyer AM, Feenstra L, Veereman-Wauters G. The complexity of feeding problems in 700 infants and young children presenting to a tertiary care institution. J Pediatr Gastroenterol Nutr. 2003 Jul 37(1):75-84.

3 Daniels $H$, Devlieger H, Minami T, Eggermont $\mathrm{E}$, Casaer P. Infant feeding and cardiorespiratory maturation. Neuropediatrics. 1990 Feb;21(1):9-10.

4 Jadcherla SR, Wang M, Vijayapal AS, Leuthner SR. Impact of prematurity and co-morbidities on feeding milestones in neonates: a retrospective study. J Perinatol. 2010 Mar; 30(3):201-8.

5 Hwang YS, Ma MC, Tseng YM, Tsai WH. Associations among perinatal factors and age of achievement of full oral feeding in very preterm infants. Pediatr Neonatol. 2013 Oct; 54(5):309-14.

6 Giannì ML, Sannino P, Bezze E, Plevani L, di Cugno N, Roggero P, et al. Effect of co-morbidities on the development of oral feeding ability in pre-term infants: a retrospective study. Sci Rep. 2015 Nov;5(1):16603.
7 Lau C. Development of Suck and Swallow Mechanisms in Infants. Ann Nutr Metab. 2015;66 Suppl 5:7-14.

8 Goyal RK, Chaudhury A. Physiology of normal esophageal motility. J Clin Gastroenterol. 2008 May-Jun;42(5):610-9.

9 Rayyan M, Omari T, Abu-Assi R, Allegaert K, Rommel N. Effect of esophageal length on high-resolution manometry metrics: extension to the neonatal population. Neurogastroenterol Motil. 2020 Feb;32(2):e13800.

10 Omari TI, Miki K, Fraser R, Davidson G, Haslam R, Goldsworthy W, et al. Esophageal body and lower esophageal sphincter function in healthy premature infants. Gastroenterology. 1995 Dec;109(6):1757-64.

11 Omari TI, Benninga MA, Barnett CP, Haslam RR, Davidson GP, Dent J. Characterization of esophageal body and lower esophageal sphincter motor function in the very premature neonate. J Pediatr. 1999 Oct;135(4):51721.

12 Jadcherla SR, Duong HQ, Hofmann C, Hoffmann R, Shaker R. Characteristics of upper oesophageal sphincter and oesophageal body during maturation in healthy human neonates compared with adults. Neurogastroenterol Motil. 2005 Oct;17(5):663-70.

13 Staiano A, Boccia G, Salvia G, Zappulli D, Clouse RE. Development of esophageal peristalsis in preterm and term neonates. Gastroenterology. 2007 May;132(5):1718-25.
14 Pena EM, Parks VN, Peng J, Fernandez SA, Di Lorenzo C, Shaker R, et al. Lower esophageal sphincter relaxation reflex kinetics: effects of peristaltic reflexes and maturation in human premature neonates. Am J Physiol Gastrointest Liver Physiol. 2010 Dec;299(6):G138695.

15 Omari TI, Miki K, Davidson G, Fraser R, Haslam R, Goldsworthy W, et al. Characterisation of relaxation of the lower oesophageal sphincter in healthy premature infants. Gut. 1997 Mar;40(3):370-5.

16 Mittal RK, Holloway RH, Penagini R, Blackshaw LA, Dent J. Transient lower esophageal sphincter relaxation. Gastroenterology. 1995 Aug;109(2):601-10.

17 van der Knaap MS, Valk J, Bakker CJ, Schooneveld M, Faber JA, Willemse J, et al. Myelination as an expression of the functional maturity of the brain. Dev Med Child Neurol. 1991 Oct;33(10):849-57.

18 Engle WA; American Academy of Pediatrics Committee on Fetus and Newborn. Age terminology during the perinatal period. Pediatrics. 2004 Nov;114(5):1362-4.

19 Rommel N, Omari T, Staunton E, French J, Davidson G. Esophageal motility patterns in children with gastro-esophageal reflux disease. Gastroenterology. 2003 Apr;124(4): A258. 
20 Singendonk MM, Ferris LF, McCall L, Seiboth G, Lowe K, Moore D, et al.; In Association with the European Society for Pediatric Gastroenterology, Hepatology, Nutrition (ESPGHAN) Pediatric Motility Network. Highresolution esophageal manometry in pediatrics: effect of esophageal length on diagnostic measures. Neurogastroenterol Motil. 2020 Jan;32(1):e13721.

21 Myers JC, Omari T. Esophageal impedance measured during peak peristaltic contraction correlates with endoscopic findings of mucosal inflammation in patients with gastroesophageal reflux symptoms. Gastroenterology. 2014 May;146(5):S752-S.

22 Mei L, Babaei A. Contractile segment impedance (CSI) during high-resolution impedance manometry highly correlates with intraluminal baseline impedance (BI), and is inversely related to esophageal acid exposure. Gastroenterology. 2018 May;154(6):S85-6.

23 Gyawali CP, Kahrilas PJ, Savarino E, Zerbib F, Mion F, Smout AJ, et al. Modern diagnosis of GERD: the Lyon Consensus. Gut. 2018 Jul; 67(7):1351-62.
24 Crist J, Gidda JS, Goyal RK. Intramural mechanism of esophageal peristalsis: roles of cholinergic and noncholinergic nerves. Proc Natl Acad Sci USA. 1984 Jun;81(11):3595-9.

25 Islami H, Shabani R, Bexheti S, Behluli I, Sukalo $A$, Raka $D$, et al. In vitro examination of ontogenesis of developing neuronal cells in vagal nuclei in medulla oblongata in newborns. Bosn J Basic Med Sci. 2008 Nov;8(4): 381-5.

26 Pereyra PM, Zhang W, Schmidt M, Becker LE. Development of myelinated and unmyelinated fibers of human vagus nerve during the first year of life. J Neurol Sci. 1992 Jul; 110(1-2):107-13.

27 Cock C, Chen CL, Lei WY, Wong MW, Besanko L, Burgstad CM, et al. 1072 - Diagnostic utility of contractile segment impedance (CSI) for the diagnosis of gastro-esophageal reflux disease (GERD). Gastroenterology. 2019 May; 156(6):S-224.
28 Jadcherla SR, Shubert TR, Malkar MB, Sitaram S, Moore RK, Wei L, et al. Gestational and postnatal modulation of esophageal sphincter reflexes in human premature neonates. Pediatr Res. 2015 Nov;78(5):540-6.

29 Kahrilas PJ, Bredenoord AJ, Fox M, Gyawali $\mathrm{CP}$, Roman S, Smout AJ, et al.; International High Resolution Manometry Working Group. The Chicago Classification of esophageal motility disorders, v3.0. Neurogastroenterol Motil. 2015 Feb;27(2):160-74.

30 Omari TI, Wauters L, Rommel N, Kritas S, Myers JC. Oesophageal pressure-flow metrics in relation to bolus volume, bolus consistency, and bolus perception. United European Gastroenterol J. 2013 Aug;1(4):249-58.

31 Omari TI, Dejaeger E, Tack J, Van Beckevoort $\mathrm{D}$, Rommel N. Effect of bolus volume and viscosity on pharyngeal automated impedance manometry variables derived for broad Dysphagia patients. Dysphagia. 2013 Jun;28(2): 146-52. 